\title{
Fatty Acyl Chain Composition in the Determination of Renal Membrane Order
}

\author{
Michael K. Hise, William W. Mantulin, and Edward J. Weinman \\ University of Texas Medical School, Department of Internal Medicine, Division of Nephrology; and The Baylor College of Medicine, \\ Department of Internal Medicine, Houston, Texas 77225
}

\begin{abstract}
The relative roles of phospholipid fatty acyl chain length and phospholipid fatty acyl chain unsaturation in the determination of rat renal brush border membrane order were examined using multilamellar liposomes. Exposure of brush border membranes to sphingomyelinase resulted in a time- and concentration-dependent decrement in sphingomyelin content. Liposomes prepared from lipid extracts of these membranes were reconstituted to defined phosphatidylcholine (PC)/sphingomyelin (SPH) ratios with pure synthetic PCs of defined chain length and degrees of unsaturation. Mixed-acid PCs from bovine liver, egg, and the rat renal brush border membrane were also examined. The steady state fluorescence anisotropy of 1,6-diphenyl-1,3,5-hexatriene (DPH) at $37^{\circ} \mathrm{C}$ was used to reflect acyl chain packing. The steady state anisotropy of DPH in liposomes isolated from the rat renal brush border membrane averaged $0.205 \pm 0.001, n=8$. When liposomes were reconstituted to PC/SPH ratios of 1.1, 1.6, and 2.4 with saturated PCs of acyl chain length 16 to 22 , differences in anisotropy between groups were not observed. However, when PCs containing unsaturated or mixed-acid fatty acyl chains were introduced, anisotropy decreased in a concentration dependent fashion. These data suggest that phospholipid fatty acyl chain unsaturation, but not acyl chain length, has a powerful influence on renal brush border membrane order and the PC/SPH ratio is an important determinant of renal membrane order by virtue of the unsaturated fatty acids normally present with these phospholipids.
\end{abstract}

\section{Introduction}

Previous studies have indicated that phospholipid fatty acyl chain motion is more restricted in brush border membranes isolated from rat or human renal cortex than in basolateral membranes isolated from those tissues $(1,2)$. Similar studies in the dog kidney have shown that the microviscosity of the brush border membrane is approximately twice that of the basolateral membrane (3). Differences seen in intact membranes in these studies were evident in liposomes prepared from lipid extracts, which suggests that the lipid composition played a dominant role in these observations. Compositional factors that are known to play a major role in the determination of natural membrane order have been reviewed (4) and studied in the rat kidney (1). Of potential importance in determining the differences between rat renal mem-

Received for publication 7 November 1984 and in revised form 18 November 1985.

J. Clin. Invest.

(c) The American Society for Clinical Investigation, Inc.

0021-9738/86/03/0768/06 \$1.00

Volume 77, March 1986, 768-773 branes are a greater phosphatidylcholine (PC) $)^{1} /$ sphingomyelin (SPH) ratio in the basolateral membrane and differences in phospholipid fatty acyl chains. The purpose of this study was to examine the relative importance of phospholipid fatty acyl chain length, acyl chain unsaturation, and the $\mathrm{PC} / \mathrm{SPH}$ ratio in the complex lipid mixture of the rat renal brush border membrane.

\section{Methods}

Membrane preparation. We used male Sprague-Dawley rats weighing 225-310 g for all studies. Animals were housed in an animal care facility and allowed free access to food and water. Rats were decapitated and their kidneys rapidly harvested and placed in iced $0.145 \mathrm{M} \mathrm{NaCl}$. The capsules were removed and the cortex was dissected on a glass plate in an ice bath. Cortices were minced with a razor and homogenized with 20 strokes of a motor-driven Dounce homogenizer using Teflon pestles. The homogenization solution contained $200 \mathrm{mM}$ mannitol, $50 \mathrm{mM}$ Tris, and $80 \mathrm{mM}$ Hepes ( $\mathrm{pH}$ 7.5). Magnesium sulfate was added to a concentration of $10 \mathrm{mM}$. Brush border membranes were isolated by standard techniques (5-7). Basolateral membranes were prepared by the method of Sacktor et. al. (8); cortical tissue was homogenized in $250 \mathrm{mM}$ sucrose and $10 \mathrm{mM}$ Tris ( $\mathrm{pH}$ 7.6). The purities of these membranes were determined by enzyme markers and were similar to values previously reported from this laboratory $(1,9)$.

In studies in which sphingomyelin degradation was carried out, brush border membrane vesicles were centrifuged at $23,000 \mathrm{~g}$ for $25 \mathrm{~min}$ at $2^{\circ} \mathrm{C}$ and resuspended in $12 \mathrm{ml}$ of buffer containing $210 \mathrm{mM}$ mannitol, $10 \mathrm{mM} \mathrm{MgCl}_{2}$, and $50 \mathrm{mM}$ Tris/Hepes (pH 7.3). The suspensions were kept on ice for $30 \mathrm{~min}$ and centrifuged as described. The vesicles were resuspended in buffer at a concentration of $4-8 \mathrm{mg} / \mathrm{ml}$.

Sphingomyelin degradation. Pure phospholipids were purchased from Supelco, Inc., Bellefonte, PA. These lipids migrated as single iodine staining spots using the two-dimensional thin layer chromatography system described below. 1,000 nmol of SPH, PC, phosphatidylethanolamine (PE), phosphatidylserine (PS), or phosphatidylinositol (PI) were transferred into $12-\mathrm{ml}$ centrifuge tubes and the organic solvents evaporated under $\mathrm{N}_{2}$. To solubilize the lipids, we added $250 \mu$ l of buffer containing $210 \mathrm{mM}$ mannitol, $10 \mathrm{mM} \mathrm{MgCl}_{2}, 50 \mathrm{mM}$ Tris/Hepes (pH 7.3) and $1 \%$ sodium deoxycholate, and the tubes were vortexed. The tubes were preincubated at $37^{\circ} \mathrm{C}$ for $10 \mathrm{~min}$ and sphingomyelinase (Bacillus cereus, Boehringer Mannheim Diagnostics, Inc., Houston, TX) was added at a concentration of $2 \times 10^{-4} \mathrm{U} / \mathrm{nmol}$ phospholipid. After addition of the enzyme, the tubes were again vortexed and incubated for $20 \mathrm{~min}$ at $37^{\circ} \mathrm{C}$. Phospholipids were extracted by adding $5 \mathrm{ml}$ of $2: 1$ chloroform/ methanol. The tubes were capped and allowed to set at $0^{\circ} \mathrm{C}$ for $1 \mathrm{~h}$, after which $1 \mathrm{ml}$ of $0.04 \mathrm{M} \mathrm{NaCl}$ was added. The tubes were vortexed and centrifuged at $5,000 \mathrm{~g}$ for $20 \mathrm{~min}$. The upper aqueous-methanolic phase and the protein interface were aspirated and the organic phase was decanted into clean tubes. The organic phase was evaporated to dryness under $\mathrm{N}_{2}$. The residue was dissolved in $500 \mu \mathrm{l}$ of 2:1 chloroform/methanol and $50 \mu \mathrm{l}$ was spotted on $\mathbf{5 0 0}$ micron silica gel layers containing $\mathbf{1 0 \%}$ magnesium acetate (Supelco, Inc.). Plates were developed in two dimensions. The first solvent system contained chloroform/methanol/

1. Abbreviations used in this paper: DPH, 1,6-diphenyl-1,3,5-hexatriene; PC, phosphatidylcholine; PE, phosphatidylethanolamine; PI, phosphatidylinositol; PS, phosphatidylserine; SPH, sphingomyelin. 
$\mathrm{NH}_{4} \mathrm{OH}(65: 25: 5, \mathrm{vol} / \mathrm{vol})$, and the second contained chloroform/methanol/ $\mathrm{H}_{2} \mathrm{O}(65: 25: 4, \mathrm{vol} / \mathrm{vol})$. Plates were developed under a nitrogen atmosphere and dried between developments in a Plexiglas tank flushed with dry nitrogen. Phospholipids were identified by exposure of the chromatograms to iodine vapors. The areas containing the phospholipids were scraped into acid-washed test tubes and the phosphorus content was determined. The recovery of authentic lipids from thin layer plates has previously been described from this laboratory (1).

In studies in which brush border membrane sphingomyelin degradation was examined as a function of time, brush border membranes containing $1,200 \mathrm{nmol}$ of SPH were suspended in $3 \mathrm{ml}$ of buffer containing $210 \mathrm{mM}$ mannitol, $10 \mathrm{mM} \mathrm{MgCl}, 50 \mathrm{mM}$ Tris/Hepes (pH 7.3) and preincubated for $10 \mathrm{~min}$ at $37^{\circ} \mathrm{C}$. Sphingomyelinase $\left(2 \times 10^{-4} \mathrm{U} /\right.$ nmol sphingomyelin) was added; the tubes were briefly vortexed and incubated at $37^{\circ} \mathrm{C}$. At 10 -min intervals, $250 \mu \mathrm{l}$ of the mixture was decanted; phospholipids were extracted in $5 \mathrm{ml}$ of 2:1 chloroform/methanol and isolated by the Folch technique (10). Phospholipid classes were separated and quantitated as described above.

Liposome preparation. Membrane vesicle suspensions were divided into two portions. One portion was exposed to sphingomyelinase (3 $\times 10^{-4} \mathrm{U} / \mathrm{nmol} \mathrm{SPH}$ ) for $25 \mathrm{~min}$ at $37^{\circ} \mathrm{C}$. Phospholipids were extracted from both preparations using the Folch technique (10). The organic phase was evaporated to dryness and the residue dissolved in 2:1 chloroform/ methanol so that the lipids associated with $1 \mathrm{mg}$ of membrane protein were in $50 \mu \mathrm{l}$ of solvent. Three distinct $\mathrm{PC} / \mathrm{SPH}$ ratios were evaluated: 1.1, 1.6, and 2.4. The total phospholipid content of the liposomes was kept constant and equivalent to brush border membrane liposomes. This was accomplished by adding phosphatidylcholine at the $\mathrm{PC} / \mathrm{SPH}$ ratios of $1.1,1.6$, and 2.4 in amounts exactly equal to the reduction in sphingomyelin at the respective ratios. The reduction in sphingomyelin and the amount of PC added were dictated by the initial values of $P C$ and SPH in the membrane. In these studies, the brush border membrane $\mathrm{PC} / \mathrm{SPH}$ ratio averaged $0.7 \pm 0.1, n=8$. To determine the amounts of extracts necessary to achieve the desired ratios, the following calculations were performed: $x \mathrm{PC}_{0}+y \mathrm{PC}_{25}=\mathrm{PC}_{1.1}$, where $x$ is a fraction of the extract prepared from membranes not exposed to enzyme, $y$ is a fraction of the extract derived from membranes exposed to sphingomyelinase for $25 \mathrm{~min}, \mathrm{PC}_{0}$ is the $\mathrm{PC}$ content of the unexposed preparation, $\mathrm{PC}_{25}$ is the $P C$ content of the extract from membranes exposed to enzyme, and $\mathrm{PC}_{1.1}$ is the PC content of the final extract that is contributed by natural membrane phosphatidylcholine at the $1.1 \mathrm{PC} / \mathrm{SPH}$ ratio. The fact that the enzyme did not affect $P C$ was verified by triplicate phosphorus analysis of the samples. Since $\mathrm{PC}_{0}=\mathrm{PC}_{25}$ and this equals $\mathrm{PC}_{1.1}$, this equation can be reduced to the form $x+y=1$ or $y=1-x$. A similar equation can be described for sphingomyelin; that is, $x \mathrm{SPH}_{0}+y \mathrm{SPH}_{25}=\mathrm{SPH}_{1.1}$, where $x$ is a fraction of the extract from unexposed membranes and $y$ is a fraction of the extract prepared from membranes previously exposed to enzyme. $\mathrm{SPH}_{0}$ is the $\mathrm{SPH}$ content of the unexposed preparation, and $\mathrm{SPH}_{25}$ is the $\mathrm{SPH}$ content of the preparation exposed to enzyme. $\mathrm{SPH}_{1.1}$ is the sphingomyelin content in the final extract at the $1.1 \mathrm{PC} / \mathrm{SPH}$ ratio. $\mathrm{SPH}_{0}$ and $\mathrm{SPH}_{25}$ were determined experimentally and $\mathrm{SPH}_{1.1}$ was derived mathematically. Since two unknowns are identified, the two equations can be solved simultaneously. Analogous equations were described for the 1.6 and the 2.4 PC/SPH ratios for each sample. The final extract volume was $50 \mu \mathrm{l}$. The portion of PC not contributed at the respective ratios by natural membrane $\mathrm{PC}$ was added to the organic extract in the form of synthetic PC (Avanti Polar Lipids, Inc., Birmingham, AL) to achieve the desired PC/SPH ratio. In some studies, PC isolated from bovine liver or egg was added. These were purchased from Avanti Polar Lipids, Inc. and migrated as single iodine staining spots using the twodimensional thin layer chromatography system described above. To examine the role of $\mathrm{PC}$ isolated from the rat renal brush border membrane, brush border membranes were extracted of their lipid content by the method of Folch (10). The organic phase was evaporated to dryness under $\mathrm{N}_{2}$ and the residue dissolved in 2:1 chloroform/methanol so that the phospholipids associated with $1 \mathrm{mg}$ of protein would be in $50 \mu \mathrm{l}$ of solvent. This amount was spotted on thin layer plates that were developed in two dimensions as described. The PC spots from multiple plates were scraped into a glass stoppered centrifuge tube and the phospholipids were extracted by adding $7 \mathrm{ml}$ of 2:1 chloroform/methanol, vortexing vigorously, and centrifuging at $7,500 \mathrm{~g}$ for $15 \mathrm{~min}$. The supernatant was decanted into a clean glass stoppered tube and the silica gel was reextracted with $7 \mathrm{ml}$ of solvent. The supernatants were combined and evaporated to dryness under $\mathrm{N}_{2}$. The lipids were dissolved in 2:1 chloroform/methanol. The purity of these lipids was examined by plating $150 \mathrm{nmol}$ (phosphorus) of the extract on silica gel layers and developing the plates in two dimensions using the solvent pair previously described by this laboratory (1). After drying, the chromatograms were exposed to iodine vapors for prolonged periods. The lipids in the extract migrated as a single iodine staining spot coincident with authentic PC. To further examine the purity of the product, $150 \mathrm{nmol}$ (phosphorus) were plated on silica gel $G$ plates and developed in two dimensions using the solvent pair described by Rouser et al. (11). Again, the product migrated as a single iodine staining spot coincident with PC. An identical procedure was used to isolate SPH from rat renal brush border membranes. Purity of this phospholipid was examined as described above and no contaminating lipids were found.

In some studies, extracts were prepared as described except that instead of adding synthetic or natural phosphatidylcholine at the $\mathrm{PC} / \mathrm{SPH}$ ratios of $1.1,1.6$, or 2.4 , SPH extracted from the rat renal brush border membrane was added in quantities that would restore the $\mathrm{PC} / \mathrm{SPH}$ ratio to its original value.

Fluorescence polarization. The well studied fluorescence probe 1,6diphenyl-1,3,5-hexatriene (DPH) was used in these studies to determine membrane order because this probe partitions equally well between fluid and gel-like phases $(12,13)$. To the final organic extract, sufficient DPH in ethanolic stock was added so that the lipid/probe ratio would be at least 500:1. The organic extracts were evaporated to dryness under $\mathrm{N}_{2}$ and residual solvents were removed by vacuum. The lipids were suspended in $5 \mathrm{ml}$ of $0.1 \mathrm{M}$ phosphate buffer $(35 \mathrm{mM} \mathrm{NaCl}, 1 \mathrm{mM} \mathrm{KCl}$, $\mathrm{pH}$ 7.4). Liposomes were prepared by vortexing in the presence of glass beads. The probe was excited at $360 \mathrm{~nm}$ and fluorescence intensities were measured using an SLM-8000 (SLM Instruments, Urbana, IL) fluorescence spectrophotometer and a cut-off filter (3-144; Corning Glass Works, Corning, NY). Light scattering was reduced to $<1 \%$ of the total signal by this filter. The cuvettes were stirred individually using a magnetic bar, and temperature was controlled by a circulating water bath. A thermocouple and a digital thermometer were used to monitor sample temperature. The steady state fluorescence anisotropy was calculated as previously described (1). Recent evidence suggests that the steady state anisotropy reflects acyl chain packing or order rather than the more dynamic property of probe rotation per se (14-16). Higher anisotropy values are associated with more ordered membranes. ${ }^{2}$

Fatty acid analysis. Phospholipids were separated using thin layer techniques. Gel containing $\sim 1 \mu \mathrm{mol}$ of PC or SPH derived from multiple plates were combined and the lipids eluted as described above. The supernatants were evaporated to dryness under nitrogen. The residues were resuspended in $2 \mathrm{ml}$ of $2: 1$ chloroform/methanol and $n$-pentadecanoic acid was added as an internal standard. Fatty acid analysis was carried out using a 5700A gas chromatograph (Hewlett-Packard Co., Palo Alto, CA) as previously described (1).

Proteins were determined by the method of Lowry et al. (17). Lipid phosphorus was determined by the method of Marinetti (18). Statistics were performed using the unpaired student's $t$ test or Tukey's multiple comparison procedure where appropriate $(19,20)$.

2. Data using the technique of electron spin resonance and either spinlabeled phosphatidylcholine in rat renal membranes (1) or spin-labeled fatty acids in the renal membranes of dog and human kidney $(2,3)$ indicate that order differs between brush border and basolateral membranes. Similar data were obtained using fluorescence polarization techniques and DPH. These findings are compatible with the notion that steady state anisotropy reflects membrane order. 


\section{Results}

As indicated in Fig. 1, exposure of pure sphingomyelin to sphingomyelinase resulted in a significant degradation of this phospholipid. A small but significant reduction was seen also in phosphatidylethanolamine when this phospholipid was exposed to enzyme. This was attributed to small amounts of contaminating phospholipases as seen in previous studies (21). The degradation of PE might be predicted since this can be a preferred substrate for phospholipase $(22,23)$. No significant reduction in PI, PS, or PC was seen. When brush border membranes were exposed to sphingomyelinase (Fig. 2), a time-dependent reduction in sphingomyelin content was observed. As shown in the figure, $\sim 70 \%$ of sphingomyelin was degraded. No further reduction in sphingomyelin content occurred despite increased time of incubation or increased enzyme quantities (data not shown). The fact that not all of the sphingomyelin was degraded suggests that a portion of sphingomyelin is not localized to the external bilayer leaflet or that some of the vesicles were inverted. Because the majority of PE is thought to be localized to the inner leaflet of the bilayer, it was of interest to see if it was susceptible to enzyme attack (24). As is evident by the data in Table I, no reduction in PE, PS, PI, or PC could be discerned when brush border membranes were exposed to sphingomyelinase. The data in Fig. 1 and Table I suggest that PE in these membranes is inaccessible to the enzyme.

Sphingomyelinase degrades sphingomyelin to phosphocholine and ceramide. Phosphocholine migrates into the upper aqueous-methanolic phase of the Folch extract. Ceramide, on the other hand, might partition into the organic phase. To investigate if degradatory products alone might effect the steady state anisotropy of DPH, we prepared liposomes for the 1.1, 1.6, and 2.4 $\mathrm{PC} / \mathrm{SPH}$ ratios, except sphingomyelin isolated from the rat renal brush border membrane was added to the extracts instead of PC. The quantities of sphingomyelin added were sufficient to make the $\mathrm{PC} / \mathrm{SPH}$ ratio identical to that of unperturbed liposomes. Therefore, the PC/SPH ratio in these liposomes did not differ from that of native brush border membrane liposomes but any degradatory products would be equivalent to liposomes prepared for the $1.1,1.6$, or $2.4 \mathrm{PC} / \mathrm{SPH}$ ratio. Anisotropy of DPH in native brush border membranes averaged $0.205 \pm 0.001$, $n=8($ mean $\pm \mathrm{SE})$. Anisotropy of DPH in liposomes preparation

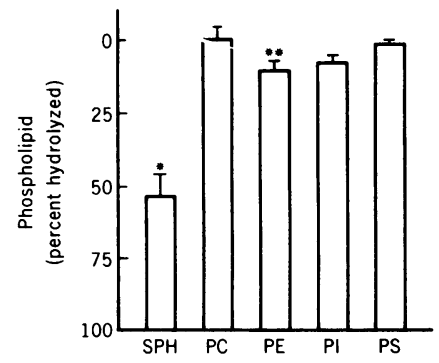

Figure 1. 1,000 nmol of pure phospholipids were individually exposed to sphingomyelinase $(2$ $\times 10^{-4} \mathrm{U} / \mathrm{nmol}$ ) for $20 \mathrm{~min}$ at $37^{\circ} \mathrm{C}$. The reaction mixture contained $210 \mathrm{mM}$ mannitol, $10 \mathrm{mM} \mathrm{MgCl}, 50 \mathrm{mM}$ Tris/ Hepes (pH 7.3), and $1 \%$ sodium deoxycholate. Lipids were extracted by the method of Folch, chromatographed using two dimension thin layer techniques, and quantitated by phosphorus analysis. Abbreviations: SPH, sphingomyelin; PC, phosphatidylcholine; PE, phosphatidylethanolamine; PI, phosphatidylinositol; PS, phosphatidylserine. Four separate preparations were studied. Data are expressed as means $\pm \mathrm{SE}$. A significant reduction in both sphingomyelin $(P<0.004)$ and phosphatidylethanolamine $(P<0.03)$ were evident.

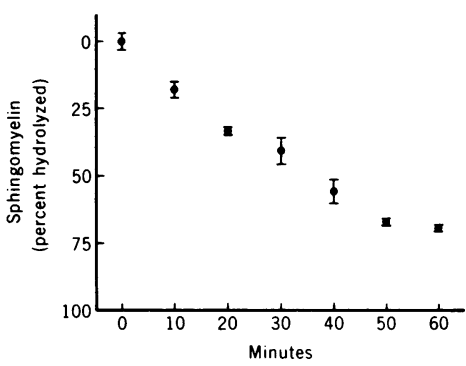

Figure 2. Rat renal brush border membranes containing $1,200 \mathrm{nmol}$ of sphingomyelin were incubated in 3 $\mathrm{ml}$ of buffer containing 210 $\mathrm{mM}$ mannitol, $10 \mathrm{mM}$ $\mathrm{MgCl}_{2}, 50 \mathrm{mM}$ Tris/Hepes (pH 7.3), and sphingomyelinase $\left(2 \times 10^{-4} \mathrm{U} / \mathrm{nmol}\right.$ sphingomyelin) at $37^{\circ} \mathrm{C}$. At 10-min intervals, a $250-\mu 1$ portion was removed and the phospholipids were extracted and quantitated. Three separate membrane preparations were studied. Data are expressed as means $\pm \mathrm{SE}$.

for the 1.1 $\mathrm{PC} / \mathrm{SPH}$ ratio but reconstituted with sphingomyelin to the original $\mathrm{PC} / \mathrm{SPH}$ ratio averaged $0.205 \pm 0.002, n=4$. Those reconstituted from the 1.6 ratio averaged $0.201 \pm 0.001, n=4$, while those reconstituted from the 2.4 ratio averaged $0.198 \pm 0.002, n=4$.

As shown in Table II, when brush border membrane liposomes were reconstituted with fully saturated PCs of chain length 16 to 22, there were no differences in anisotropy between groups at defined $\mathrm{PC} / \mathrm{SPH}$ ratios. The fact that values were greater than control liposomes and liposomes reconstituted with sphingomyelin suggests that saturated PCs have a rigidifying effect. To examine whether differences in chain length could be discerned in liposomes containing unsaturated fatty acids, we examined PCs containing fatty acids with one double bond (Table III). The double bond of dimyristoleoyl and dioleoyl PC is at carbon 9. Despite concentration-dependent decrements in anisotropy, no independent influence of chain length was evident at any of the $\mathrm{PC} / \mathrm{SPH}$ ratios examined.

The role of unsaturation in membrane order was examined by reconstituting liposome with PCs containing unsaturated fatty acids. As can be seen by the data in Table III, reconstitution with PCs containing unsaturated fatty acids caused a concentration-dependent decrement in the steady state anisotropy at $37^{\circ} \mathrm{C}$ over the range of $\mathrm{PC} / \mathrm{SPH}$ ratios examined. These findings indicate that the presence of fatty acids with unsaturated bonds decrease order. No differences were apparent, however, between

Table I. Phospholipid Composition of Rat Cortical Brush Border Membranes Exposed to Sphingomyelinase

\begin{tabular}{lllll}
\hline Minutes & PC & PE & PS & PI \\
\hline 0 & $98.2 \pm 6.8$ & $108.4 \pm 7.6$ & $56.9 \pm 4.1$ & $14.5 \pm 1.4$ \\
25 & $94.8 \pm 5.6$ & $112.2 \pm 8.1$ & $59.3 \pm 4.2$ & $13.4 \pm 2.0$ \\
\hline
\end{tabular}

Intact brush border membranes were exposed to sphingomyelinase $\left(3 \times 10^{-4} \mathrm{U} / \mathrm{nmol}\right.$ sphingomyelin) for 0 or $25 \mathrm{~min}$. Phospholipids were extracted from these membranes and the major classes were separated using two-dimensional thin layer chromatography; phospholipids were quantitated by phosphorus analysis. Data are expressed as nanomoles of lipid per milligram protein \pm SE. Abbreviations: PC, phosphatidylcholine; PE, phosphatidylethanolamine; PS, phosphatidylserine; PI, phosphatidylinositol. Differences between individual phospholipid classes were not evident when the 0 - and 25-min time points were compared. Six separate membrane preparations were studied. 
Table II. Steady State Fluorescence Anisotropy of DPH in Brush Border Membrane Liposomes Containing Fully Saturated Phosphatidylcholines

\begin{tabular}{|c|c|c|c|c|}
\hline \multirow[b]{2}{*}{ PC } & & \multicolumn{3}{|l|}{$\mathrm{PC} / \mathrm{SPH}$ ratio } \\
\hline & & 1.1 & 1.6 & 2.4 \\
\hline Dipalmitoyl & $16: 0$ & $0.215 \pm 0.001$ & $0.218 \pm 0.001$ & $0.215 \pm 0.001$ \\
\hline Distearoyl & $18: 0$ & $0.215 \pm 0.001$ & $0.216 \pm 0.004$ & $0.220 \pm 0.002$ \\
\hline Diarachidoyl & $20: 0$ & $0.215 \pm 0.002$ & $0.219 \pm 0.001$ & $0.220 \pm 0.002$ \\
\hline Dibehenoyl & $22: 0$ & $0.213 \pm 0.001$ & $0.213 \pm 0.003$ & $0.212 \pm 0.003$ \\
\hline
\end{tabular}

Studies were performed at $37^{\circ} \mathrm{C}$. The number before the colon denotes the acyl chain length; the number after the colon indicates the number of unsaturated bonds. Four separate sets of liposomes were analyzed at each ratio. Values for each experiment are the means of triplicate determinations. Data are expressed as means \pm SE. Differences between groups were not apparent at defined PC/SPH ratios.

liposomes containing dioleoyl, dilinoleoyl, dilinolenoyl, or diarchidonoyl PC, which suggests that the number of unsaturated bonds has little effect.

Natural membranes contain phospholipids that are a combination of saturated and unsaturated fatty acids $(25,26)$. Liposomes were reconstituted to defined PC/SPH ratios using phosphatidylcholines from natural sources. As can be seen by the data in Table IV, the addition of natural PCs showed a pattern similar to that of unsaturated PCs, that is, a progressive decrease in anisotropy as the PC/SPH ratio is increased. Furthermore, the natural membranes PCs were similar to one another.

Since one of the major compositional differences between brush border and basolateral membranes is the PC/SPH ratio, it was of interest to examine the $\mathrm{PC} / \mathrm{SPH}$ ratio at 1.6 since this approximates the basolateral membrane (1). Anisotropy of DPH in basolateral membrane liposomes averaged $0.179 \pm 0.007, n$ $=4$, while liposomes reconstituted to the $1.6 \mathrm{PC} / \mathrm{SPH}$ with brush border membranes PC averaged $0.188 \pm 0.003, n=4$ (Table IV).

Table III. Steady State Fluorescence Anisotropy of DPH in Brush Border Membrane Liposomes Containing Progressively Unsaturated Phosphatidylcholines

\begin{tabular}{lllll}
\hline & \multicolumn{3}{l}{ PC/SPH ratio } \\
\cline { 3 - 5 } PC & & 1.1 & 1.6 & 2.4 \\
\hline Distearoyl & $18: 0$ & $0.215 \pm 0.001^{*}$ & $0.216 \pm 0.004^{*}$ & $0.220 \pm 0.002^{*}$ \\
Dimyristoleoyl & $14: 1$ & $0.203 \pm 0.002 \ddagger$ & $0.193 \pm 0.002$ & $0.185 \pm 0.003$ \\
Dioleoyl & $18: 1$ & $0.197 \pm 0.002 \ddagger$ & $0.193 \pm 0.002$ & $0.186 \pm 0.002$ \\
Dilinoleoyl & $18: 2$ & $0.194 \pm 0.003 \ddagger$ & $0.189 \pm 0.003$ & $0.180 \pm 0.003$ \\
Dilinolenoyl & $18: 3$ & $0.195 \pm 0.002 \ddagger$ & $0.189 \pm 0.002$ & $0.179 \pm 0.002$ \\
Diarachidonoyl & $20: 4$ & $0.195 \pm 0.001 \ddagger$ & $0.187 \pm 0.002$ & $0.179 \pm 0.003$
\end{tabular}

Studies were done at $37^{\circ} \mathrm{C}$. The number before the colon denotes the acyl chain length; the number after the colon indicates the number of unsaturated bonds. Four separate sets of liposomes were analyzed at each PC/SPH ratio. Values for each experiment are the means of triplicate determinations. Data are expressed as means \pm SE. The data for distearoyl PC are reproduced from Table II for comparison.

* $P \leq 0.01$ when compared with individual unsaturated PC's at the indicated ratios.

$\ddagger P \leq 0.01$ when compared with values at the $2.4 \mathrm{PC} / \mathrm{SPH}$ ratio.
Table IV. Steady State Fluorescence Anisotropy of DPH in Brush Border Membrane Liposomes Containing Mixed-acid Phosphatidylcholines from Natural Sources

\begin{tabular}{llll}
\hline \multirow{4}{*}{ PC } & \multicolumn{3}{l}{ PC/SPH ratio } \\
\cline { 2 - 4 } & 1.1 & 1.6 & 2.4 \\
\hline Distearoyl & $0.215 \pm 0.001^{*}$ & $0.216 \pm 0.004^{*}$ & $0.220 \pm 0.002^{*}$ \\
Egg & $0.198 \pm 0.001 \ddagger$ & $0.190 \pm 0.001$ & $0.184 \pm 0.001$ \\
Bovine liver & $0.198 \pm 0.001 \ddagger$ & $0.188 \pm 0.003$ & $0.184 \pm 0.002$ \\
BBM & $0.194 \pm 0.003 \ddagger$ & $0.188 \pm 0.003$ & $0.179 \pm 0.002$
\end{tabular}

Studies were done at $37^{\circ} \mathrm{C}$. Four separate sets of liposomes were analyzed at each point. Values for each experiment are the means of triplicate determinations. Data are expressed as means $\pm S E$. The data for distearoyl PC are reproduced from Table II for comparison.

* $P \leq 0.01$ when compared with individual mixed-acid PC's at the indicated ratios.

$\ddagger P \leq 0.01$ when compared with values at the $2.4 \mathrm{PC} / \mathrm{SPH}$ ratio.

To determine the degree of unsaturation of PC and SPH, we isolated these lipids from both the brush border and the basolateral membrane by thin layer techniques, and we determined fatty acid profiles by gas chromatography. As shown in Table $\mathrm{V}$, phosphatidylcholine contains greater mole percentages of the unsaturated fatty acids oleic, linoleic, and arachidonic. Phosphatidylcholine also contains a greater mole percentage of palmitic acid but no long-chain lignoceric acid was identified. Identical data were seen in both the brush border and the basolateral membrane. The combined mole percentage of unsaturated fatty acids in brush border PC averaged $40.6 \% \pm 3.1 \%$, while that in

Table V. Phospholipid Fatty Acyl Chain Composition

\begin{tabular}{|c|c|c|c|c|c|}
\hline \multirow[b]{2}{*}{ Fatty acid } & & \multicolumn{2}{|c|}{ Phosphatidylcholine } & \multicolumn{2}{|c|}{ Sphingomyelin } \\
\hline & & BBM & BLM & BBM & BLM \\
\hline Myristic & $14: 0$ & $2.0 \pm 0.4$ & $1.8 \pm 0.2$ & $2.8 \pm 0.4$ & $2.8 \pm 0.2$ \\
\hline Palmitic & $16: 0$ & $26.0 \pm 0.7 \ddagger$ & $25.1 \pm 0.7 \ddagger$ & $20.0 \pm 0.8$ & $20.6 \pm 0.3$ \\
\hline Stearic & 18:0 & $29.1 \pm 1.5$ & $28.3 \pm 1.8$ & $31.9 \pm 2.1$ & $34.9 \pm 0.6$ \\
\hline $\begin{array}{l}\text { Oleic, } \\
\text { Linoleic }\end{array}$ & $\begin{array}{l}18: 1^{*} \\
18: 2\end{array}$ & $7.8 \pm 0.5 \S$ & $8.9 \pm 0.5 \S$ & $1.3 \pm 0.5$ & $2.5 \pm 0.4$ \\
\hline Arachidic & $20: 0$ & $2.4 \pm 0.3$ & $2.0 \pm 0.3$ & $4.0 \pm 1.0$ & $3.4 \pm 0.4$ \\
\hline Arachidonic & $20: 4$ & $32.8 \pm 1.9^{\prime \prime}$ & $34.0 \pm 2.6^{\| \prime}$ & $24.6 \pm 2.5$ & $27.2 \pm 0.7$ \\
\hline Lignoceric & $24: 0$ & $0 \ddagger$ & $0 \ddagger$ & $15.5 \pm 3.6$ & $8.6 \pm 1.0$ \\
\hline
\end{tabular}

Data are expressed as mole percent \pm SE. Four separate experiments were performed in each group. The number before the colon indicates the acyl chain length; the number after the colon denotes the number of unsaturated bonds.

* The data for oleic and linoleic acids are combined. Statistical comparisons of individual fatty acids are between phosphatidylcholine and sphingomyelin within a defined membrane.

$\ddagger P<0.01$.

$\S P<0.005$.

II $P<0.05$. 
brush border SPH averaged $25.9 \% \pm 2.3 \%(n=4, P<.01)$. Similarly, the mole percentage of unsaturated fatty acids in PC isolated from the basolateral membrane was $42.9 \% \pm 2.9 \%$, while that in sphingomyelin was $29.7 \% \pm 1.0 \%(n=4, P<.01)$.

\section{Discussion}

Although the exact physiologic role of membrane physical properties has not been explicitly defined, a number of experimental observations in prokaryotes and mammalian systems have indicated that order has an important effect on the activity of membrane bound proteins (27-29). The following factors, mainly in artificial systems, have been identified as being major determinants of the physical properties of membranes: the lipid to protein ratio; the ratio of cholesterol to phospholipid; the ratio of phosphatidylcholine to sphingomyelin; and the length and degree of unsaturation of the fatty acyl chains. The relative importance of these factors in natural membranes, in general, and in brush border membranes of the kidney, in particular, are unknown. The first aim of the present studies was to examine the relative roles of acyl chain length and acyl chain unsaturation in the determination of the physical properties of the rat renal brush border membrane. In addition, these studies allowed some insight into the influence of the $\mathrm{PC} / \mathrm{SPH}$ ratio as a determinant of membrane order. The second aim of the present investigations was to determine the reasons for the differences in the physical properties between the brush border membrane and the basolateral membrane of the rat kidney.

The experimental approach used was to degrade brush border membrane sphingomyelin with sphingomyelinase because of the known specificity of this enzyme. Liposomes were reconstituted to a constant cholesterol/phospholipid ratio by the addition of phosphatidylcholines of known fatty acid composition. Thus, it was possible to examine a range of PC/SPH ratios that encompassed values previously found in the basolateral membrane of the rat kidney (1).

The products of sphingomyelin degradation are phosphocholine and ceramide. The following lines of evidence would indicate that ceramide did not affect the interpretation of the results. The addition of exogenous ceramide (bovine brain; $\mathrm{Su}$ pelco, Inc.) in amounts predicted to be generated at the $2.4 \mathrm{PC} /$ SPH ratio did not affect the anisotropy of DPH (data not shown). Moreover, at any given PC/SPH ratio, the amount of ceramide would be a constant and would not negate comparisons between the groups. The other reaction product, phosphocholine, migrates to the upper aqueous-methanolic phase of the Folch extract and, thus, would not be expected to provide a source of interference in these studies. Finally, reconstitution of liposomes with sphingomyelin produced anisotropy values that were only slightly decreased from control.

In the first series of studies (Table II), we examined the effects of added PCs containing fully saturated fatty acids of varying chain lengths. The choice of chain lengths was chosen to reflect those found in the brush border membrane of the rat kidney (1). In comparison with liposomes reconstituted to the normal $\mathrm{PC} / \mathrm{SPH}$ ratio of 0.7 , anisotropy was higher in each group. However, there were no differences between groups containing different acyl chains at defined PC/SPH ratios. To determine if differences might be apparent if unsaturation were introduced into the acyl chain, we studied PCs with one double bond at carbon 9 (Table III). Although the influence of unsaturated fatty acids was evident, there was no independent effect of chain length. Taken together, these findings would indicate that the length of the fatty acyl chain has little or no effect on the physical properties of these membranes. In the second series of experiments, we examined the influence of fatty acyl chain unsaturation on bulk membrane order. The presence of an unsaturated bond is believed to disrupt the packing of the acyl chains in membranes by introducing a "kink" in the fatty acid. These fatty acids occupy a greater partial specific volume and disorder the packing array. In contrast to the influence of chain length, the introduction of fatty acids with unsaturated bonds results in a progressive decrease in anisotropy (decrease in order) as the PC/SPH ratio is increased (Table III). By comparing the effect on anisotropy of unsaturated PCs dioleoyl, dilinoleoyl, and dilinolenoyl with that of the saturated PC distearoyl, the independent influence of unsaturation becomes apparent. Furthermore, by comparing the individual groups at varying $\mathrm{PC} / \mathrm{SPH}$ ratios, the effect of the PC/SPH ratio is evident. Of particular interest is that over the range examined, the presence of multiple unsaturated bonds confers no further changes in membrane order. This is compatible with previous studies in artificial systems $(30,31)$.

The phospholipids of natural membranes, for the most part, contain one saturated and one unsaturated fatty acid $(25,26)$. It seemed reasonable, therefore, to examine the effects of natural membrane PCs. As shown in Table IV, reconstitution with PCs from egg, bovine liver, or PC purified from the rat renal brush border membrane results in a progressive decrease in anisotropy as the $\mathrm{PC} / \mathrm{SPH}$ ratio is increased. The PC/SPH ratio of 1.6 was of particular interest since this is the ratio of the basolateral membrane of the rat kidney. The data indicate that the PC/SPH ratio explains, in part, the differences in physical properties between brush border and basolateral membranes.

Taken together, these data predict that differences in fatty acyl chain unsaturation might be important in determining the differences in physical properties between phosphatidylcholine and sphingomyelin. Studies by Lentz et al. (32) indicate that acyl chain composition may, in fact, be the most important determinant of the differences in physical properties between these lipids. Studies in human kidney have indicated that sphingomyelin contains less unsaturated fatty acids than does PC (33). To examine this issue in renal membranes, we examined the fatty acid profiles of PC and SPH in both the brush border and the basolateral membrane. As shown in Table V, phosphatidylcholine contains greater mole percentages of the unsaturated fatty acids oleic, linoleic, and arachidonic when compared with sphingomyelin. The fatty acid profiles of phosphatidylcholine were not different when the brush border and the basolateral membranes were compared. Similar data was obtained for sphingomyelin.

In summary, phospholipid fatty acyl chain unsaturation exerts a powerful influence on renal membrane order. The phosphatidylcholine/sphingomyelin ratio is an important determinant of membrane order, most likely, by virtue of the fatty acids normally contained in these lipids. The phosphatidylcholine/ sphingomyelin ratio is an important determinant of the differences in physical properties between brush border and basolateral membranes of the rat kidney. 


\section{Acknowledgments}

The authors wish to thank Pramod Mehta for excellent technical assistance, and Paula J. Lewis for transcribing the manuscript. This work was funded in part by National Institutes of Health grant HL-27104.

\section{References}

1. Hise, M. K., W. W. Mantulin, and E. J. Weinman. 1984. Fluidity and composition of brush border and basolateral membranes from rat kidney. Am. J. Physiol. 247(Renal Fluid Electrolyte Physiol. 16):F434F439.

2. Le Grimellec, C., S. Carriere, J. Cardinal, and M.-C. Giocondi. 1983. Fluidity of brush border and basolateral membranes from human kidney cortex. Am. J. Physiol. 245(Renal Fluid Electrolyte Physiol. 14): F227-F231.

3. Le Grimellec, C., M.-C. Giocondi, B. Carriere, S. Carriere, and J. Cardinal. 1982. Membrane fluidity and enzyme activities in brush border and basolateral membranes of the dog kidney. Am. J. Physiol. 242(Renal Fluid Electrolyte Physiol. 11):F246-F253.

4. Shinitzky, M., and P. Henkart. 1979. Fluidity of cell membranescurrent concepts and trends. Int. Rev. Cytol. 60:121-145.

5. Aronson, P. S. 1978. Energy dependence of phlorizin binding to isolated renal microvillus membranes. J. Membr. Biol. 42:81-98.

6. Blomstedt, J. W., and P. S. Aronson. 1980. pH gradient-stimulated transport of urate and p-aminohippurate in dog renal microvillus membrane vesicles. J. Clin. Invest. 65:931-934.

7. Kahn, A. M., S. Branham, and E. J. Weinman. 1983. Mechanism of urate and $p$-aminohippurate transport in rat renal microvillus membrane vesicles. Am. J. Physiol. 245(Renal Fluid Electrolyte Physiol. 14): F151-F158.

8. Sacktor, B., I. L. Rosenbloom, C. T. Liang, and L. Cheng. 1981. Sodium gradient and sodium plus potassium gradient-dependent L-glutamate uptake in renal basolateral membrane vesicles. J. Membr. Biol. 60:63-71.

9. Kahn, A. M., S. Branham, and E. J. Weinman. 1984. Mechanism of $\mathrm{L}$-malate transport in rat renal basolateral membrane vesicles. Am. J. Physiol. 246(Renal Fluid Electrolyte Physiol. 15):F779-F784.

10. Folch, J., M. Lees, and G. H. Sloane Stanley. 1957. A simple method for the isolation and purification of total lipids from animal tissues. J. Biol. Chem. 226:497-509.

11. Rouser, G., C. Galli, E. Lieber, M. L. Blank, and O. S. Privett. 1964. Analytical fractionation of complex lipids mixtures: DEAE cellulose column chromatography combined with quantitative thin layer chromatography. J. Am. Oil Chem. Soc. 41:836-840.

12. Lentz, B. R., Y. Barenholz, and T. E. Thompson. 1976. Fluorescence depolarization studies of phase transitions and fluidity in phospholipid bilayers. 2. Two-component phosphatidylcholine liposomes. Biochemistry. 15:4529-4537.

13. Jahnig, F., H. Vogel, and L. Best. 1982. Unifying description of the effect of membrane proteins on lipid order. Verification for the melittin/dimyristoylphosphatidylcholine system. Biochemistry. 21:67906798.

14. Jahnig, F. 1979. Structural order of lipids and proteins in membranes: Evaluation of fluorescence anisotropy data. Proc. Natl. Acad. Sci. USA. 76:6361-65.
15. Van Blitterswijk, W. J., R. P. Van Hoeven, and B. W. Van Der Meer. 1981. Lipid structural order parameters (reciprocal of fluidity) in biomembranes derived from steady-state fluorescence polarization measurements. Biochim. Biophys. Acta. 644:323-332.

16. Pottel, H., W. Van Der Meer, and W. Herreman. 1983. Correlation between the order parameter and the steady-state fluorescence anisotropy of 1,6-diphenyl-1,3,5-hexatriene and an evaluation of membrane fluidity. Biochim. Biophys. Acta. 730:181-186.

17. Lowry, O. H., N. J. Rosebrough, A. L. Farr, and R. J. Randall. 1951. Protein measurement with the Folin phenol reagent. J. Biol. Chem. 193:265-275.

18. Marinetti, G. V. 1962. Chromatographic separation, identification, and analysis of phospholipids. J. Lipid Res. 3:1-20.

19. Snedecor, G. W., and W. G. Cochran. 1967. Statistical Methods, 6th ed. Iowa State University Press, Ames, IA. 59-62.

20. Kleinbaum, D. G., and L. L. Kupper. Applied regression analysis and other multivariable methods. Duxbury Press, North Scituate, MA. 264-271.

21. Ikezawa, H., M. Mori, T. Ohyabu, and R. Taguchi. 1978. Studies on sphingomyelinase of bacillus cereus I. purification and properties. Biochim. Biophys. Acta. 528:247-256.

22. Weglick, W. B., M. Waite, P. Sisson, and S. B. Shohet. 1971. Myocardial phospholipase A of microsomal and mitochondrial fractions. Biochim. Biophys. Acta. 231:512-519.

23. Natori, Y., K. Karasawa, H. Arai, Y. Tamori-Natori, and S. Nojima. 1983. Partial purification and properties of phospholipase $A_{2}$ from rat liver mitochondria. J. Biochem. 93:631-637.

24. Bergelson, L. D., and L. I. Barsukov. 1977. Topological asymmetry of phospholipids in membranes. Science (Wash. DC). 197:224-230.

25. Van Golde, L. M., and L. L. M. van Deenen. 1966. The effect of dietary fat on the molecular species of lecithin from rat liver. Biochim. Biophys. Acta. 125:496-509.

26. Kuksis, A., W. C. Breckenridge, L. Marai, and O. Stachnyk. 1969. Molecular species of lecithins of rat heart, kidney, and plasma. $J$. Lipid Res. 10:25-32.

27. Amatruda, J. M., and E. D. Finch. 1979. Modulation of hexose uptake and insulin action by cell membrane fluidity. J. Biol. Chem. 254: 2619-2625.

28. Sandermann, H., Jr. 1978. Regulation of membrane enzymes by lipids. Biochim. Biophys. Acta. 515:209-237.

29. McElhaney, R. N. 1982. Effects of membrane lipids on transport and enzymatic activities. In Current Topics in Membranes and Transport. S. Razin and S. Rottem, editors. Academic Press, Inc., New York, 317380.

30. Evans, R. W., and J. Tinoco. 1978. Monolayers of sterols and phosphatidylcholines containing a 20-carbon chain. Chem. Phys. Lipids. 22:207-220.

31. Stubbs, C. D., T. Kouyama, K. Kinosita, Jr., and A. Ikegami. 1981. Effect of double bonds on the dynamic properties of the hydrocarbon region of lecithin bilayers. Biochemistry. 20:4257-4262.

32. Lentz, B. R., M. Hoechli, and Y. Barenholz. 1981. Acyl chain order and lateral domain formation in mixed phosphatidylcholinesphingomyelin multilamellar and unilamellar vesicles. Biochemistry. 20: 6803-6809.

33. Druilhet, R. E., M. L. Overturf, and W. M. Kirkendall. 1975. Structure of neutral glycerides and phosphoglycerides of human kidney. Int. J. Biochem. 6:893-901. 